\title{
Higher integrability of the gradient in degenerate elliptic equations
}

Pierre Dreyfuss (pierre.dreyfuss@unifr.ch )

Mathematics Department, University of Fribourg, Pérolles, CH-1700 Fribourg, Switzerland.

\begin{abstract}
We prove that under some global conditions on the maximum and the minimum eigenvalue of the matrix of the coefficients, the gradient of the (weak) solution of some degenerate elliptic equations has higher integrability than expected. Technically we adapt the Giaquinta-Modica regularity method in some degenerate cases. When the dimension is two, a consequence of our result is a new Hölder continuity result for the weak solution.
\end{abstract}

Keywords: Degenerate elliptic equations, regularity of solutions, weights

1991 Mathematics SubjectClassification: 35J70, 35B65

\section{Introduction}

Let $\Omega$ be an open bounded set in $\mathbb{R}^{n}$ ( $n$ equals 2 or 3 ), with a Lipschitz boundary. We consider a linear, second order, self adjoint, degenerate elliptic equation with a homogeneous Dirichlet boundary condition :

(P) $\left\{\begin{array}{l}\mathcal{L} u:=-\sum_{i, j=1}^{n} \frac{\partial}{\partial x_{i}}\left(a_{i j}(x) \frac{\partial u}{\partial x_{j}}\right)=f \text { in } \Omega, \\ u=0 \text { on } \partial \Omega .\end{array}\right.$

Here $f: \Omega \rightarrow \mathbb{R}$ is a given function and $\mathcal{A}(x):=\left[a_{i j}(x)\right]_{i, j=1, . . n}$ is a given symmetric matrix with measurable coefficients. We assume that $\mathcal{A}$ is positive definite almost everywhere in $\Omega$, and we denote by $\lambda(x)$, $\Lambda(x)$ its minimum and maximum eigenvalues. It follows that for all $\eta, \theta \in \mathbb{R}^{n}$ and a.e $x \in \Omega$ we have :

$$
\begin{aligned}
& \langle\mathcal{A}(x) \eta, \theta\rangle \leq \Lambda(x)|\eta||\theta|, \\
& \langle\mathcal{A}(x) \eta, \eta\rangle \geq \lambda(x)|\eta|^{2},
\end{aligned}
$$

* This work is supported by the Swiss National Science Foundation under the Grant Number 200020-100051/1.

(C) 2006 Kluwer Academic Publishers. Printed in the Netherlands.

cor_article.tex; 20/04/2006; $11: 32 ;$ p.2 
where $\langle$,$\rangle denotes the scalar product in \mathbb{R}^{n}$.

In this paper we will study some questions about existence, uniqueness and regularity of weak solution for problem $(\mathrm{P})$. We will also give a regularity result for the weak solution of a class of non-linear degenerate problems which include $(\mathrm{P})$.

\subsection{Mathematical BaCKGround}

When $\lambda$ may vanish or $\Lambda$ may be undbounded then $\mathcal{L}$ is called degenerate operator. We will always assume in the following that $\lambda$ is strictly positive almost everywhere and $\Lambda$ is finite almost everywhere. These assumptions are not sufficient to analyse problem (P) and therefore we will also assume :

$$
\begin{aligned}
\lambda & \in L^{1}(\Omega), \\
\lambda^{-1} & \in L^{1}(\Omega), \\
\frac{\Lambda}{\lambda} & \in L^{\infty}(\Omega) .
\end{aligned}
$$

For $p \geq 1$, we denote by $L^{p}(\lambda, \Omega)$ the weighted Lebesgue space defined by

$$
L^{p}(\lambda, \Omega)=\left\{u: \Omega \rightarrow \mathbb{R} \text { measurable and s.t } \int_{\Omega} \lambda(x)|u(x)|^{p} d x<\infty\right\}
$$

and equipped with the norm

$$
\|u\|_{L^{p}(\lambda, \Omega)}=\left(\int_{\Omega} \lambda(x)|u(x)|^{p} d x\right)^{1 / p} .
$$

Hence $L^{p}(\lambda, \Omega)$ is a Banach space. Moreover assumption (3) ensures that $\mathcal{D}(\Omega) \subset L^{p}(\lambda, \Omega)$ with dense injection.

Under the assumptions (3)-(5), the natural functional setting for problem $(\mathrm{P})$ is given by the following weighted Sobolev spaces :

$$
\begin{aligned}
W & =\left\{u \in W^{1,1}(\Omega):\|u\|_{\lambda}<\infty\right\}, \\
W_{0} & =\left\{u \in W_{0}^{1,1}(\Omega):\|u\|_{\lambda}<\infty\right\}, \\
H & =\overline{\mathcal{C}^{\infty}(\bar{\Omega})} W \\
H_{0} & =\overline{\mathcal{C}_{c}^{\infty}(\Omega)} W
\end{aligned}
$$


where we have used the notation

$$
\|u\|_{\lambda}^{2}:=\|u\|_{L^{2}(\lambda, \Omega)}^{2}+\|\nabla u\|_{L^{2}(\lambda, \Omega)}^{2} .
$$

The spaces $W, W_{0}, H$ and $H_{0}$ are Hilbert spaces and we have $H \subset W \subset$ $\mathcal{D}^{\prime}(\Omega)$ and $H_{0} \subset W_{0}$. Notice that assumption (4) is quite necessary. If we remove it then $W_{0}$ need not be complete and the gradient of a function in $H_{0}$ need not be uniquely defined (see [40] and [31] Proposition $1.2)$.

Let $\mathcal{B}$ denote the bilinear form on $\mathcal{D}(\Omega)$ associated to $\mathcal{L}$ :

$$
\mathcal{B}(u, \varphi):=\int_{\Omega}\langle A(x) \nabla u(x), \nabla \varphi(x)\rangle d x .
$$

By using assumption (1) together with (5) we obtain

$$
|\mathcal{B}(u, \varphi)| \leq C\|u\|_{\lambda}\|\varphi\|_{\lambda} \quad \forall u, \varphi \in W,
$$

where $C:=\left\|\frac{\Lambda}{\lambda}\right\|_{L^{\infty}(\Omega)}$. The bilinear form $\mathcal{B}$ is then continuous on $W$ and we can consider two natural notions of weak solutions for Problem $(\mathrm{P})$ :

W-solution : Let $f \in W_{0}^{\prime}$. A function $u \in W_{0}$ is called a W-solution of $(\mathrm{P})$ if it verifies

$$
\mathcal{B}(u, \varphi)=\langle f, \varphi\rangle_{W_{0}^{\prime}, W_{0}} \quad \forall \varphi \in W_{0} .
$$

H-solution : Let $f \in H_{0}^{\prime}$. A function $u \in H_{0}$ is called a H-solution of $(\mathrm{P})$ if it verifies

$$
\mathcal{B}(u, \varphi)=\langle f, \varphi\rangle_{H_{0}^{\prime}, H_{0}} \quad \forall \varphi \in H_{0} .
$$

Note that in general, for $n \geq 2, H_{0} \neq W_{0}$ (see [40]), and even for smooth second member, we can obtain a W-solution and a H-solution for Problem (P) that are not equal (see [40] Proposition 1.1, and [41]). When we have the equality $H=W$, which also implies $H_{0}=W_{0}$ (see [31], remark 1.5), we say that $\lambda$ is regular. Sufficient conditions ensuring that a weight $\lambda$ is regular were established in [40] and [15]. An exact characterisation of regular weights is not known. In the sequel we will assume that

$$
\lambda \text { is a regular weight. }
$$

It follows that the two notions of $\mathrm{W}$-solution and $\mathrm{H}$-solution are the same notion and we call it weak solution. Clearly in this case we also 
have $H_{0}^{\prime}=W_{0}^{\prime}$. Remark that, by definition, $\mathcal{D}(\Omega)$ is dense in $H_{0}$ so that $H_{0}^{\prime}$ can be identified with a subspace of $\mathcal{D}^{\prime}(\Omega)$. In fact (see [29] p.8), a distribution $T \in \mathcal{D}^{\prime}(\Omega)$ is in $H_{0}^{\prime}$ if and only if it can be represented (in general non uniquely) as

$$
\langle T, \varphi\rangle=\int_{\Omega}\left(f_{0}(x) \lambda(x) \varphi(x)+\lambda(x)\langle f(x), \nabla \varphi(x)\rangle\right) d x \quad \forall \varphi \in \mathcal{D}(\Omega),
$$

with $f_{0} \in L^{2}\left(\lambda^{-1}, \Omega\right)$ and $f \in\left(L^{2}\left(\lambda^{-1}, \Omega\right)\right)^{n}$.

\subsection{MAin Results}

Instead of (3)-(4), we will consider the following stronger assumptions for $\lambda$ :

$$
\begin{aligned}
\lambda^{-1} & \in L^{\infty}(\Omega), \\
\lambda & \in W^{1,1}(\Omega), \\
\text { there exists } & \sigma>2 \text { s. t. } \int_{\Omega} \frac{|\nabla \lambda|^{\sigma}}{\lambda^{\frac{3 \sigma}{2}}}<\infty .
\end{aligned}
$$

Notice that condition (16) is equivalent to $\lambda^{-1 / 2} \in W^{1, \sigma}(\Omega)$. Finally, we will consider $\lambda$ in the following class $\mathcal{K}$ of weights :

$$
\mathcal{K}:=\{\lambda>0 \text { a.e in } \Omega \text { and it satisfies (13), (14) - (16) }\} .
$$

We will consider problem $(\mathrm{P})$ with a second member satisfying

$$
f \in W^{-1, p}(\Omega), \quad \text { with } p>n .
$$

Notice that, when $\lambda$ is in the class $\mathcal{K}$ and $p>n$, we have

$$
H_{0}=W_{0} \subset H_{0}^{1}(\Omega) \subset W_{0}^{1, p^{\prime}}(\Omega) .
$$

It follows that if $f$ satisfies (17) then $f \in H_{0}^{\prime}$.

Our main result is the following :

THEOREM 1. Assume that $\lambda$ is in the class $\mathcal{K}$ and (5) is fulfilled. Then, for any $f \in H_{0}^{\prime}$, there exists a unique weak solution $u$ for problem $(P)$, satisfying :

$$
\|u\|_{\lambda} \leq C\|f\|_{H_{0}^{\prime}}
$$


If in addition $f$ satisfies (17) then $u \in L^{\infty}(\Omega)$ and

$$
\|u\|_{L^{\infty}(\Omega)} \leq C\|f\|_{-1, p} .
$$

Moreover there exist $\varepsilon>0$ and $C<\infty$ depending only on $\Omega, f$ and $\lambda$, such that

$$
\|\nabla u\|_{\left(L^{2+\varepsilon}(\Omega)\right)^{n}} \leq C .
$$

A consequence of Theorem 1 (see [5] Theorem IX.12 p.166) is :

COROLLARY 2. When the dimension $n$ equals two and $f$ satisfies (17) then the weak solution u given by Theorem 1 is Hölder continuous.

The result presented in the last part of Theorem 1, and in the Corollary 2 remains valid for a general class of non-linear degenerate problems. In fact, we can consider the following problems :

$$
\left(\mathrm{P}^{\prime}\right) \quad\left\{\begin{array}{l}
-\operatorname{div} \mathcal{A}(x, u, \nabla u)=f(x, u, \nabla u) \quad \text { in } \Omega, \\
u=0 \text { on } \partial \Omega,
\end{array}\right.
$$

where, $\mathcal{A}: \Omega \times \mathbb{R} \times \mathbb{R}^{n} \rightarrow \mathbb{R}^{n}$ and $f: \Omega \times \mathbb{R} \times \mathbb{R}^{n} \rightarrow \mathbb{R}$ are Caratheodory functions that satisfy the following growth and coercivity conditions :

$$
\begin{aligned}
|\mathcal{A}(x, t, \eta)| & \leq \mu_{1}\left(\Lambda(x)|\eta|+\varphi_{1}(x)\right), \\
\mathcal{A}(x, t, \eta) \eta & \geq \mu_{2}\left(\lambda(x)|\eta|^{2}-\varphi_{2}(x)\right), \\
|f(x, t, \eta)| & \leq \mu_{3}\left(\Lambda(x)|\eta|^{\alpha}+\varphi_{3}(x)\right) .
\end{aligned}
$$

Here, $\varphi_{i}, i=1,2,3$, are positive functions satisfying

$$
\frac{\varphi_{1}^{2}}{\lambda^{2}}, \frac{\varphi_{2}^{2}}{\lambda^{2}} \in L^{s}(\Omega) \text { and } \frac{\varphi_{3}}{\lambda} \in L^{s}(\Omega) \text { for some } s>1,
$$

$\alpha<2$ is a positive number and $\mu_{1}, \mu_{3} \geq 0, \mu_{2}>0$ are allowed to depend on $t$.

We have :

THEOREM 3. Assume that $\lambda \in \mathcal{K},(5)$ holds true and $\mathcal{A}, f$ are Caratheodory functions satisfying (21)-(23). Assume moreover that $u \in H_{0} \cap L^{\infty}(\Omega)$ is a weak solution of problem ( $\left.P^{\prime}\right)$. Then there exists $\varepsilon>0$ such that $\int_{\Omega}|\nabla u|^{2+\varepsilon}<\infty$. In particular, for $n$ equals two, the function $u$ is Hölder continuous. 


\section{Remarks:}

(i) The results in Theorem 1, Corollary 2 and Theorem 3 remain valid if $\lambda$ is in the following class $\mathcal{K}^{\prime}$ which includes $\mathcal{K}$ :

$\mathcal{K}^{\prime}:=\left\{\begin{array}{l}\lambda>0 \text { a.e. in } \Omega \text { and it is regular. Moreover } \lambda=\delta \rho, \text { with } \\ \delta \in L^{\infty}(\Omega), \delta^{-1} \in L^{\infty}(\Omega) \text { and } \rho \text { satifies }(14)-(16) .\end{array}\right\}$

(ii) The assumption $\lambda$ regular can be removed in $\mathcal{K}$ or $\mathcal{K}^{\prime}$. In this situation we have to consider $\mathrm{W}$-solutions instead of weak solutions for problem $(\mathrm{P})$ and we recover the results in Theorem 1, Corollary 2 and Theorem 3.

\subsection{Discussion on the literature}

Degenerate problems like (P) have been extensively studied for many years.

In general, the existence of a weak solution is obtained after proving a Poincaré inequality (see [29], [37] and [31]). In particular the first part of Theorem 1, i.e., the existence and uniqueness of the weak solution $u$ satisfying (18) is an application of [29] Corrolary 3.5.

For some studies about the question of the boundedness of $u$ we can consult [29], [31] and [11]. In Theorem 1, the property (19) is an application of [29] Theorem 7.1.

The Hölder continuity of the weak solution, or the higher integrability of its gradient (in the sense of (20)) have also studied been studied for many years. The first situation considered was the case of uniform ellipticity, that is when we have

$$
\text { (UE) } \quad \lambda \in L^{\infty}(\Omega), \lambda^{-1} \in L^{\infty}(\Omega) .
$$

In this situation, the Hölder continuity of the weak solution $u$ (there is only one notion because $\lambda$ is regular, see [40]) was established in the works of DeGiorgi, Nash and Moser (see [9, 27, 28, 30]), without restriction on the dimension.

On the other hand, a result of higher integrability for $\nabla u$ was obtained by Boyarski and Meyers (see [3, 24] and [13, 25, 17]).

These results were later generalised in numerous works. The principal generalisation of (UE) we want to point out is the following :

$$
\text { (M) } \quad \lambda \in \mathcal{A}_{2} \text {. }
$$


Here $\mathcal{A}_{2}$ is the Muckhenhoupt class of order two, i.e. $\lambda \in \mathcal{A}_{2}$ means

$$
\sup _{\text {balls } B \subset \Omega} \int_{B} \lambda f_{B} \lambda^{-1}<\infty
$$

where we have used the notation :

$$
f_{B} g(y) d y:=|B|^{-1} \int_{B} g(y) d y .
$$

We can see that (UE) implies (M). In this case $\lambda$ is regular, and Hölder continuity of the weak solution $u$ (again without restriction on the dimension) was established by Fabes, Koenig and Serapioni (see [11] and $[1,39])$. Moreover we also have a higher integrability result for $\nabla u$ (see [35]).

We will see in Section 2 that $\lambda \in \mathcal{K}$ does not imply (M). It follows that Theorem 1, Corollary 2 and Theorem 3 cannot be deduced from the results in [35] or [11].

Notice also that, contrary to $(\mathrm{M})$, our assumptions in $\mathcal{K}$ are of global nature. This is an important advantage for some applications of the higher integrability result, as will be explained in the next paragraph. Under the assumption $\lambda \in \mathcal{K}$, we will obtain the higher integrability result for $\nabla u$ by using a method inspired by the works of GiaquintaModica (see [17]) and Stredulinsky (see [35]). In the situation they consider (case (UE) or (M)), the following three important properties hold:

(I) The measure $\lambda d x$ is doubling, i.e.

$$
\sup _{B(x, 2 r) \subset \Omega} \frac{\int_{B(x, 2 r)} \lambda(y) d y}{\int_{B(x, r)} \lambda(y) d y}<\infty .
$$

(II) $\lambda$ is regular.

(III) Uniform Poincaré-Sobolev inequality on the balls, i.e. for all $B(x, r) \subset$ $\Omega$ :

$\left(\int_{B(x, r)}|u(y)-\bar{u}|^{2} \lambda(y) d y\right)^{2} \leq C\left(\int_{B(x, r)}|\nabla u(y)|^{\frac{2 n}{2+n}} \lambda(y) d y\right)^{\frac{2+n}{2 n}}, \quad \forall u \in H$

where $C$ is a constant, and $\bar{u}:=\frac{\int_{B(x, r)} u(y) \lambda(y) d y}{\int_{B(x, r)} \lambda(y) d y}$. 
The properties (I) and (III) are necessary for their techniques to work. In fact, they employ certain test functions in the weak formulation and, by using (I) and (III) they obtain a weak-reverse Hölder inequality for $\nabla u$. After this, the higher integrability result for $\nabla u$ follows from a certain version of the Gehring lemma. The point is that, when $\lambda \in \mathcal{K}$, then the properties (I) and (III) need not hold (see the counterexamples in Section 3). Nevertheless we obtain the higher integrability of the gradient of $u$ by using different test functions in the weak formulation. Notice that there exist relations between the properties (I)-(III) (see $[31,20,2])$.

In some cases, a higher integrability for the gradient can be obtained from interpolation theory (see [6]). Similar results can be established for parabolic equations (see $[16,22]$ ).

\subsection{Applichtions of the Results}

Differential problems like (P) arrise in many physical models such as oceanography (see $[4,23]$ ), turbulent fluid flows (see [15]), induction heating (see [8]) and electrochemical problems (see [14]). The knowledge of some regularity results for problem $(\mathrm{P})$ is useful for the analysis of these physical models. In particular, a higher integrability result for the gradient of the weak solution of problem $(\mathrm{P})$ would be useful for the analysis of the models studied in $[8,14,15]$. In fact, in these works, the problem analyzed is to find two scalar functions $u, h: \Omega \rightarrow \mathbb{R}$ vanishing on $\partial \Omega$ and such that:

$$
\begin{aligned}
& -\operatorname{div}(r(h) \nabla u)=f \quad \text { in } \mathcal{D}^{\prime}(\Omega), \\
& -\operatorname{div}(b(h) \nabla h)=r(h)|\nabla u|^{2} \quad \text { in } \mathcal{D}^{\prime}(\Omega),
\end{aligned}
$$

where $f \in L^{2}(\Omega)$ and $r, b \in \mathcal{C}^{1}(\mathbb{R})$ are given. One way to solve Problem (24)-(25) is to decuple the two equations. First we solve (24) with a given $h=\bar{h}$, in some Sobolev space. This subproblem is in fact a particular case of Problem $(\mathrm{P})$ where we have $\mathcal{A}(x)=r(\bar{h}) I d$. In a second step, we want to solve (25) with a second member $r(\bar{h})|\nabla u|^{2}$ which is known, but only a priori to be in $L^{1}(\Omega)$. This latter fact creates difficulties for the subsequent analysis. The situation would be more favorable if the second member $r(\bar{h})|\nabla u|^{2}$ would be an element of $L^{s}(\Omega)$, for some $s>1$. This property should be obtained in some cases, if we can apply a result like higher integrability for $\nabla u$. For instance, this is the case if we assume that $r$ and $r^{-1}$ are in $L^{\infty}(\mathbb{R})$. It is then possible to apply the Meyers result (see [7]). However, the assumption $r, r^{-1} \in L^{\infty}(\mathbb{R})$ doest not always have physical relevance (see $\left.[7,15]\right)$.

cor_article.tex; 20/04/2006; $11: 32 ;$ p.9 
Under more restrictive conditions on $r$ we would apply the Stredulinsky result, but here the difficulty is to find precisely what these conditions are. In fact, we have to ensure that $r(\bar{h}) \in \mathcal{A}_{2}$, which is not easy if we recall the definition of an $\mathcal{A}_{2}$-weight. Here our regularity results presented in Theorem 1 and 3 are easier to use.

\subsection{Organization of the Paper}

In Section 2 we will present the proof of Theorem 1. The higher integrability result for the gradient of the weak solution is obtained from a weak-reverse Hölder inequality. The method is inspirated by the works of Giaquinta-Modica (case (UE), see [17]) and Stredulinsky (case (M), see [35]), but the originality resides in a special choice of test functions. The reason is that, contrary to the case where $\lambda$ is in the class (M), if $\lambda \in \mathcal{K}$ then the measure $\lambda d x$ need not be doubling (see the counter examples in Section 3) and we do not need to have a uniform Poincaré-Sobolev inequality on the balls in $H_{0}$. Theses two properties are necessary in the technique of Giaquinta-Modica and Stredulinsky. With a particular choice of test functions we are able to overcome this difficulty.

Theorem 3 is proved in the same manner. We also give some indications concerning the remarks at the end of Paragraph 1.2.

In Section 3, we construct in dimension two and three a weight $\lambda \in \mathcal{K}$ which is not in (M). In dimension three the example presented is particulary instructive. It is apparently close to satisfying the condition (UE) but we will prove that in fact it does not satisfy (M). Moreover this weight does not have bounded mean oscillations.

\section{The proofs}

\subsection{The First Part of the Proof of Theorem 1}

Assume that $\lambda$ and $\Lambda$ satisfy the assumptions in Theorem 1 . Let $f \in H_{0}^{\prime}$. The weak formulation for problem (P) consists in finding $u \in H_{0}\left(=W_{0}\right)$ such that:

$$
\mathcal{B}(u, \varphi)=\langle f, \varphi\rangle \quad \forall \varphi \in H_{0}
$$


Recall first that the bilinear form $\mathcal{B}$ is continuous, as seen in (12).

The first part of Theorem 1 is a consequence of Corrolary 3.5 p.22 and Theorem 7.1 p.49 in [29]. In fact the assumption (15) implies that $\lambda \in L^{\frac{n}{n-1}}(\Omega)$, and by using (14) we can see that the condition (3.2)' in [29] p.21 is fullfilled (for $n=2$ take $p=2, s=2, t=2$ and for $n=3$ take $p=2, s=3 / 2, t=\infty)$.

Hence, from Corollary 3.5 in [29] we obtain :

$$
\|\nabla \varphi\|_{L^{2}(\lambda, \Omega)} \geq C_{1}\|\varphi\|_{\lambda} \quad \forall \varphi \in H_{0}
$$

where $C_{1}>0$ is a constant depending only on $\Omega$ and $\lambda$.

It follows that the bilinear form $\mathcal{B}$ defined by (11) is coercive on $H_{0}$. Then, by the Lax-Milgram theorem, we obtain a unique solution for (26), with the estimate

$$
\|u\|_{\lambda} \leq C_{2}\|f\|_{H_{0}^{\prime}}, \quad C_{2}=C_{2}\left(C_{1}\right) .
$$

Let us now consider $f$ satisfying (17). By using [5], proposition IX.20 p.175, we obtain the existence of a function $g$ as follows :

$$
\begin{aligned}
g \in\left(L^{p}(\Omega)\right)^{n}:\langle f, \varphi\rangle_{W^{-1, p}, W^{1, p^{\prime}}} & =\int_{\Omega}\langle g(x), \nabla \varphi(x)\rangle d x, \\
& \forall \varphi \in W_{0}^{1, p^{\prime}}(\Omega) .
\end{aligned}
$$

Recall that $\lambda^{-1} \in L^{\infty}(\Omega)$ and thus $g \in\left(L^{p}\left(\lambda^{-1}, \Omega\right)\right)^{n}$. We can now use Theorem 7.1 in [29] p.49 (take $s=n /(n-1), t=\infty$ and use assumption (17)). We obtain $u \in L^{\infty}(\Omega)$ and

$$
C_{4}:=\|u\|_{L^{\infty}(\Omega)} \leq C_{3}\|f\|_{-1, p} .
$$

Here, $C_{3}=C_{3}\left(C_{2}, \Omega, \lambda\right)$.

This proves the first part of Theorem 1 .

For the second part of the theorem, we will use a technique inspired by the works of Giaquinta and Modica (see [17] and [32]). We will obtain the higher integrability of the gradient of $u$ from a weak reverse Hölder inequality. The major tool is the Proposition 1.1 p.122 in [17]. Notice that this proposition is a refinement of the Gehring lemma (see [18]). Other versions of the Gehring lemma were established in [35, 21, 26].

Let $Q_{R_{0}}$ denote a $n$-cube, parallel to the coordinate axis and such that

$$
\bar{\Omega} \subset Q_{R_{0}} \quad \text { and } \quad \operatorname{dist}\left(\partial Q_{R_{0}}, \partial \Omega\right)=R_{1}>0 .
$$


Let $x \in Q_{R_{0}}$. For $r>0$, we denote by $Q_{r}(x)$ the $n$-cube centered in $x$, parallel to the coordinate axis and with side length equal $2 r$, that is :

$$
Q_{r}(x)=\left\{y \in \mathbb{R}^{n}:\left|y_{i}-x_{i}\right|<r\right\} .
$$

In the sequel we will consider the following bound for $r$ :

$$
r<\frac{1}{2} \operatorname{dist}\left(x, \partial Q_{R_{0}}\right)
$$

This condition ensures that $Q_{2 r}(x) \subset Q_{R_{0}}$. We next consider the cube $Q_{\frac{3 r}{2}}(x)$. We have three possibilities :

i) $\quad Q_{\frac{3 r}{2}}(x) \cap \Omega=\varnothing$

ii) $\quad Q_{\frac{3 r}{2}}(x) \cap\left(Q_{R_{0}} \backslash \Omega\right)=\varnothing$

iii) $\quad Q_{\frac{3 r}{2}}(x) \cap \Omega \neq \varnothing \quad$ and $\quad Q_{\frac{3 r}{2}}(x) \cap\left(Q_{R_{0}} \backslash \Omega\right) \neq \varnothing$.

For any function $\zeta$ defined on $\Omega$, we denote by $\tilde{\zeta}$ its extension on $Q_{R_{0}}$ defined by :

$$
\tilde{\zeta}=\left\{\begin{array}{l}
\zeta(x) \text { for } x \in \Omega \\
0 \text { for } x \in Q_{R_{0}} \backslash \Omega .
\end{array}\right.
$$

Let $u$ be the weak solution for problem $(\mathrm{P})$, and $\tilde{u}$ its extension on $Q_{R_{0}}$. We set

$$
\begin{aligned}
k & :=\frac{|\nabla u||\nabla \lambda|}{\lambda}+\frac{|\nabla u||g|}{\lambda}+\frac{|\nabla \lambda||g|}{\lambda^{2}}+\frac{|g|^{2}}{\lambda^{2}}, \\
q & :=\frac{2 n}{n+2} .
\end{aligned}
$$

In order to apply the Proposition 1.1 in [17], we will prove the following

LEMMA 4. There exists a positive constant $M$, depending only on $f, \lambda$ and $\Omega$ such that

$$
\underset{Q_{r}(x)}{f}|\nabla \tilde{u}|^{2} d y \leq M\left\{\left(\underset{Q_{2 r}(x)}{f}|\nabla \tilde{u}|^{q} d y\right)^{2 / q}+\underset{Q_{2 r}(x)}{f \tilde{k}} d y\right\},
$$

for each $x \in Q_{R_{0}}$ and $r \geq 0$ satisfying (32).

Let $x \in Q_{R_{0}}$ and $r \geq 0$ satisfy (32). We will prove that (35) holds true in each of the cases i), ii) and iii). 
In the first case i), we have $Q_{\frac{3 r}{2}}(x) \cap \Omega=\varnothing$ and then inequality (35) is trivial since we have $\tilde{u} \equiv 0$ on $Q_{r}(x)$.

It remains to establish (35) in the cases ii) and iii). This is the aim of the next two paragraphs.

\subsection{The Weak Reverse Hölder Inequality in the CASE II)}

Here we have $Q_{\frac{3 R}{2}}(x) \cap\left(Q_{R_{0}} \backslash \Omega\right)=\varnothing$. We will obtain the inequality (35) by using an appropriate test function in the weak formulation (26). Namely, we set

$$
\varphi=\frac{u-\bar{u}}{\lambda} \psi^{2}
$$

where $\bar{u}$ is the mean integral of $u$ over the ball $B_{\frac{3 r}{2}}(x)$ (which is contained in $\left.Q_{\frac{3 r}{2}}(x)\right)$ and $\psi$ is a cut-off function satisfying :

$$
\begin{aligned}
\psi & \in \mathcal{C}_{c}^{1}\left(B_{\frac{3 r}{2}}(x)\right), \\
0 & \leq \psi \leq 1 \text { and } \psi \equiv 1 \text { in } Q_{r}(x), \\
\mid \nabla & \psi \mid \leq \frac{C_{5}}{r}
\end{aligned}
$$

Here $C_{5}$ denotes a constant independent of $r$.

Remark that we can write $\varphi=\varphi_{1} \varphi_{2}$, with $\varphi_{1}:=(u-\bar{u}) \varphi$ and $\varphi_{2}:=\frac{\psi}{\lambda}$. By using the assumptions (14)-(16) together with the fact that $u \in W_{0} \cap$ $L^{\infty}(\Omega)$ we can see that each of the two functions $\varphi_{1}$ and $\varphi_{2}$ is an element of the space $H_{0}^{1}(\Omega) \cap L^{\infty}(\Omega)$. It follows that $\varphi \in H_{0}^{1}(\Omega) \cap L^{\infty}(\Omega)$ (see [5] proposition IX.4 p.155). In particular $\varphi \in W_{0}^{1,1}(\Omega)$ and $\int_{\Omega} \lambda|\varphi|^{2}<\infty$. In order to verify that $\varphi$ is an admissible test function for (26), it is sufficient to check that $\int_{\Omega} \lambda|\nabla \varphi|^{2}<\infty$.

To prove this, we first calculate the expression for the gradient of $\varphi$. We obtain :

$$
\nabla \varphi=\frac{1}{\lambda} \nabla u \psi^{2}-(u-\bar{u}) \frac{\nabla \lambda}{\lambda^{2}} \psi^{2}+\frac{2}{\lambda} \psi \nabla \psi(u-\bar{u}) .
$$

By using the assumptions for $\lambda$ together with the property $u \in W_{0} \cap$ $L^{\infty}(\Omega)$, we can verify that each of the three terms in the right hand side of $(40)$ are in the space $L^{2}(\lambda, \Omega)$. Consequently $\varphi$ is an admissible test function. 
We now test the equation (26) with $\varphi$. By using the expression of $\nabla \varphi$ given in (40), we obtain :

$$
\begin{aligned}
I & :=\int_{\Omega} \frac{\psi^{2}}{\lambda}\langle\mathcal{A}(x) \nabla u, \nabla u\rangle=\underbrace{\int_{\Omega} \frac{u-\bar{u}}{\lambda^{2}} \psi^{2}\langle\mathcal{A}(x) \nabla u, \nabla \lambda\rangle}_{:=I I} \\
& -\underbrace{\int_{\Omega}^{\Omega} 2 \psi \frac{u-\bar{u}}{\lambda}\langle\mathcal{A}(x) \nabla u, \nabla \psi\rangle}_{:=I I}+\underbrace{\int_{\Omega}^{\Omega} \frac{\psi^{2}}{\lambda}\langle g, \nabla u\rangle}_{:=I V} \\
& -\underbrace{\int_{\Omega}^{\Omega} \frac{u-\bar{u}}{\lambda^{2}} \psi^{2}\langle g, \nabla \lambda\rangle}_{:=V}+\underbrace{\int_{\Omega} 2 \psi}_{:=V I} \frac{u-\bar{u}}{\lambda}\langle g, \nabla \psi\rangle
\end{aligned}
$$

We estimate the term I by using (2) :

$$
I \geq \int_{\Omega}|\nabla u|^{2} \psi^{2} .
$$

By using (1) together with (5) and (30), we obtain :

$$
|I I| \leq \int_{\Omega} \frac{|u-\bar{u}|}{\lambda^{2}} \psi^{2} \Lambda|\nabla u \nabla \lambda| \leq C_{6}\left(\left\|\frac{\Lambda}{\lambda}\right\|_{L^{\infty}}, C_{4}\right) \int_{B_{\frac{3 r}{2}}(x)} \frac{|\nabla u \nabla \lambda|}{\lambda} .
$$

In order to estimate the third term, we use again (1) together with the Young inequality. We obtain :

$$
|I I I| \leq \frac{1}{2} \int_{\Omega}|\nabla u|^{2} \psi^{2}+\frac{C_{7}}{r^{2}} \int_{B_{\frac{3 r}{2}(x)}}|u-\bar{u}|^{2} .
$$

Here $C_{7}=C_{7}\left(\left\|\frac{\Lambda}{\lambda}\right\|_{L^{\infty}}, C_{4}, C_{5}\right)$.

The terms $I V$ and $V$ can be estimated by employing the property (30). We obtain :

$$
|I V|+|V| \leq C_{8} \int_{B_{\frac{3 r}{2}}(x)} \frac{|\nabla u||g|}{\lambda}+\frac{|\nabla \lambda||g|}{\lambda^{2}}, \quad C_{8}=C_{8}\left(C_{4}\right) .
$$

For the last term in (41) we use the Young inequality and property (39) to obtain :

$$
|V I| \leq C_{9}\left(\frac{1}{r^{2}} \int_{B_{\frac{3 r}{2}}(x)}|u-\bar{u}|^{2}+\int_{B_{\frac{3 r}{2}}(x)} \frac{|g|^{2}}{\lambda^{2}}\right), \quad C_{9}=C_{9}\left(C_{5}\right) .
$$


At this point, from (41), we can deduce

$$
\frac{1}{2} \int_{\Omega}|\nabla u|^{2} \psi^{2} \leq C_{10}\left(\frac{1}{r^{2}} \int_{B_{\frac{3 r}{2}}(x)}|u-\bar{u}|^{2}+\int_{B_{\frac{3 r}{2}}(x)} k\right),
$$

with $C_{10}=\operatorname{Max}\left(C_{6}, C_{7}+C_{9}, C_{8}\right)$.

We next use the property (38), and we divide (42) by $\left|B_{\frac{3 r}{2}}(x)\right|$. We have :

$$
f_{Q_{r}(x)}|\nabla u|^{2} \leq C_{11}\left(\frac{1}{r^{2}} \underset{B_{\frac{3 r}{2}}(x)}{f}|u-\bar{u}|^{2}+\underset{B_{\frac{3 r}{2}}(x)}{f} k\right), \quad C_{11}=C_{11}\left(C_{10}, n\right) .
$$

Recall now that $u \in H_{0}^{1}(\Omega)$ and thus (see [10], Theorem2 p.141) there exists $C_{12}=C_{12}(\Omega)$ such that

$$
\frac{1}{r^{2}} \underset{B_{\frac{3 r}{2}}(x)}{f}|u-\bar{u}|^{2} \leq C_{12}\left(\underset{B_{\frac{3 r}{2}}(x)}{f}|\nabla u|^{q}\right)^{2 / q}
$$

Note also that $B_{\frac{3 r}{2}}(x)$ is included in $Q_{\frac{3 r}{2}}(x)$, and the Lebesgue measure of these sets is comparable. We then get :

$$
f_{Q_{r}(x)}|\nabla u|^{2} \leq M\left(\left(\underset{Q_{\frac{3 r}{2}}(x)}{f}|\nabla u|^{q}\right)^{2 / q}+\underset{Q_{\frac{3 r}{2}}(x)}{f} k\right), \quad M=M\left(C_{11}, C_{12}\right) .
$$

and inequality (35) follows.

\subsection{The Weak Reverse Hölder inequality in the CASE IiI)}

Here the cube $Q_{\frac{3 r}{2}}(x)$ intersects $\partial \Omega$ and we have to slighty modify the technique in order to obtain (35).

Let

$$
\varphi=\frac{u}{\lambda} \psi^{2}
$$

where $\psi$ is a cut-off function verifying (37)-(39). The expression of $\nabla \varphi$ is now given by :

$$
\nabla \varphi=\frac{\nabla u}{\lambda} \psi^{2}+\frac{2}{\lambda} \psi \nabla \psi u-u \psi^{2} \frac{\nabla \lambda}{\lambda^{2}} .
$$


By using the same arguments as in Paragraph 2.2, we can verify that $\varphi \in W_{0}$.

We then test the equation (26) with $\varphi$, and, instead of (42), we now obtain :

$$
\int_{Q_{r}(x) \cap \Omega}|\nabla u|^{2} \leq C_{10}^{\prime}\left(\frac{1}{r^{2}} \int_{B_{\frac{3 r}{2}}(x) \cap \Omega}|u|^{2}+\int_{B_{\frac{3 r}{2}}(x) \cap \Omega} k\right) .
$$

Let us consider the extension $\tilde{u}$ of $u$, and the cube $Q_{2 r}(x)$ (included in $\left.Q_{R_{0}}\right)$. We have $\tilde{u} \equiv 0$ in $Q_{2 r}(x) \backslash \Omega$. Recall that we have assumed $\partial \Omega$ to be Lipschitz, which implies that $\left|Q_{2 r}(x) \backslash \Omega\right| \geq \gamma\left|Q_{2 r}(x)\right|$ for some $\gamma>0$ independently of $r$. Moreover, we clearly have $\tilde{u} \in H^{1}\left(Q_{2 r}(x)\right)$. It then follows, by using [17] Proposition p.153 and (45), that :

$$
\int_{Q_{2 r}(x)}|\nabla \tilde{u}|^{2} \leq C_{11}^{\prime}\left(\frac{1}{r^{2}}\left(\int_{Q_{2 r}(x)}|\nabla \tilde{u}|^{q}\right)^{2 / q}+\int_{Q_{2 r}(x)} \tilde{k}\right) .
$$

By dividing this inequality by $\left|Q_{r}(x)\right|$, we obtain (35). In fact, $\left|Q_{r}(x)\right|$ is comparable to $Q_{2 r}(x)$ and also comparable to $\left(1 / r^{2}\right)\left|Q_{r}(x)\right|^{2 / q}$. This ends the proof of Lemma 4.

\subsection{The Conclusion of the Proof of Theorem 1}

We set

$$
\begin{aligned}
h & :=|\nabla \tilde{u}|^{q}, \\
l & :=\tilde{k}^{q / 2},
\end{aligned}
$$

where $k$ is the function defined in (33) and $q$ is the number given in (34).

With these notations, the inequality (35) can be written as :

$$
\underset{Q_{r}(x)}{f} h^{2 / q} \leq M\left(\left(\underset{Q_{2 r}(x)}{f} h\right)^{2 / q}+\underset{Q_{2 r}(x)}{f} l^{2 / q}\right) .
$$

Note also that $2 / q=(n+2) / n>1$.

At this point, we can use Proposition 1.1 p.122 in [17]. We obtain the existence of a constant $C_{13}=C_{13}(M, q, n)$ and of $\varepsilon=\varepsilon(M, q, n)>0$ 
such that, for all $x \in Q_{R_{0}}$ and all $r<(1 / 2) \operatorname{dist}\left(x, \partial Q_{R_{0}}\right)$ the following holds :

$$
\left(\underset{Q_{r}(x)}{f} h^{\chi}\right)^{1 / \chi} \leq C_{13}\left(\left(\underset{Q_{2 r}(x)}{f} h^{2 / q}\right)^{q / 2}+\left(\underset{Q_{2 r}(x)}{f} l^{\chi}\right)^{1 / \chi}\right),
$$

where $\chi:=2 / q+\varepsilon$.

Let now $R^{*}>0$ be given by

$$
R^{*}:=\frac{R_{1}}{3}=\frac{1}{3} \operatorname{dist}\left(\partial \Omega, \partial Q_{R_{0}}\right) .
$$

Notice that, for every $x \in \bar{\Omega}$, the number $R^{*}$ satisfies the condition (32). Since $\bar{\Omega}$ is compact, we have :

$$
\bar{\Omega}=\cup_{i=1}^{m} Q_{R^{*}}\left(x_{i}\right) \cap \bar{\Omega},
$$

where $x_{1}, x_{2}, . ., x_{m}$ are some points in $\bar{\Omega}$.

By applying (49) we obtain :

$$
\left(\int_{\Omega} h^{\chi}\right)^{1 / \chi} \leq C_{14}\left(\left(\int_{\Omega} h^{2 / q}\right)^{q / 2}+\left(\int_{\Omega} l^{\chi}\right)^{1 / \chi}\right),
$$

where $C_{14}=C_{14}\left(C_{13}, m, R^{*}\right)$.

Recalling the definitions (46), (47) we have :

$$
h^{\chi}=|\nabla u|^{2+q \varepsilon}, h^{2 / q}=|\nabla u|^{2}, l^{\chi}=k^{1+q \varepsilon / 2} \quad \text { on } \Omega .
$$

In order to conclude the proof we have then to show that $k \in L^{\beta}(\Omega)$ for some $\beta>1$. We have :

$$
k=\underbrace{\frac{|\nabla u||\nabla \lambda|}{\lambda}}_{:=k_{1}}+\underbrace{\frac{|\nabla u||g|}{\lambda}}_{:=k_{2}}+\underbrace{\frac{|\nabla \lambda||g|}{\lambda^{2}}}_{:=k_{3}}+\underbrace{\frac{|g|^{2}}{\lambda^{2}}}_{:=k_{4}} .
$$

By using the assumption $\lambda^{-1 / 2} \in W^{1, \sigma}(\Omega)$ we can see that $k_{1} \in L^{\beta_{1}}(\Omega)$, with $\beta_{1}=(2 \sigma) /(2+\sigma)>1$. In fact :

$$
\begin{aligned}
\left\|k_{1}\right\|_{L^{\beta_{1}(\Omega)}} & =\left(\int_{\Omega} \lambda^{\beta_{1} / 2}|\nabla u|^{\beta_{1}} \frac{|\nabla \lambda|^{\beta_{1}}}{\lambda^{\frac{3 \beta_{1}}{2}}}\right)^{1 / \beta_{1}} \leq\left(\int_{\Omega} \lambda|\nabla u|^{2}\right)^{1 / 2}\left(\int_{\Omega} \frac{|\nabla \lambda|^{\sigma}}{\lambda^{\frac{3 \sigma}{2}}}\right)^{1 / \sigma} \\
& \leq\|u\|_{\lambda}\left\|\lambda^{-1 / 2}\right\|_{1, \sigma}<\infty .
\end{aligned}
$$

We now use (29) to see that $k_{2}, k_{3} \in L^{\beta_{2}}(\Omega)$ with $\beta_{2}=(2 p) /(2+p)>1$

$$
\begin{aligned}
\left\|k_{2}\right\|_{L^{\beta_{2}(\Omega)}} & \leq\left\|\lambda^{-1}\right\|_{L^{\infty}(\Omega)}^{3 / 2}\|u\|_{\lambda}\|g\|_{\left(L^{p}(\Omega)\right)^{n}}<\infty, \\
\left\|k_{3}\right\|_{L^{\beta_{2}(\Omega)}} & \leq\left\|\lambda^{-1}\right\|_{L^{\infty}(\Omega)}^{1 / 2}\left\|\lambda^{-1 / 2}\right\|_{1,2}\|g\|_{\left(L^{p}(\Omega)\right)^{n}}<\infty .
\end{aligned}
$$


Finally we can schow that $k_{4} \in L^{\beta_{3}}(\Omega)$, with $\beta_{3}=p / 2>1$ :

$$
\left\|k_{4}\right\|_{L^{\beta_{3}(\Omega)}} \leq\left\|\lambda^{-1}\right\|_{L^{\infty}(\Omega)}^{2}\|g\|_{\left(L^{p}(\Omega)\right)^{n}}^{2}<\infty .
$$

Hence $k \in L^{\beta}(\Omega)$, with $\beta=\min \left(\beta_{1}, \beta_{2}, \beta_{3}\right)>1$. Let $\varepsilon_{1}=2(\beta-1) / q>$ 0 . We have proved that there exists $\varepsilon \in\left(0, \varepsilon_{1}\right]$ such that

$$
\left(\int_{\Omega}|\nabla u|^{2+\varepsilon^{\prime}}\right)^{\frac{1}{2+\varepsilon^{\prime}}} \leq C_{15}\left(C_{14},\|u\|_{\lambda},\|k\|_{L^{\beta}}\right)<\infty
$$

where $\varepsilon^{\prime}:=(2 n \varepsilon) /(n+2)>0$.

We have thus proved Theorem 1

\subsection{The PRoOF OF THEOREM 3 AND INDICATIONS FOR IMPLEMENTING THE REMARKS IN PARAGRAPH 1.2}

Theorem 3 can be proved by using the same technique as for the last part of Theorem 1. We can carry over the arguments presented in Paragraphs 2.2, 2.3 and 2.5 with only slight modifications. In the sequel we will indicate the modifications needed.

Let $u \in H_{0} \cap L^{\infty}(\Omega)$ be a weak solution of problem ( $\left.\mathrm{P}^{\prime}\right)$, that is :

$$
\int_{\Omega}\langle\mathcal{A}(x, u, \nabla u), \nabla \varphi(x)\rangle d x=\int_{\Omega} f(x, u, \nabla u) \varphi(x) d x, \quad \forall \varphi \in H_{0} .
$$

Here $\mathcal{A}: \Omega \times \mathbb{R} \times \mathbb{R}^{n} \rightarrow \mathbb{R}^{n}$ and $f: \Omega \times \mathbb{R} \times \mathbb{R}^{n} \rightarrow \mathbb{R}$ are Caratheodory functions that satisfy $(21)-(22)$. We recall that the parameters $\mu_{i}$ in (21)-(22) are allowed to depend on $t$. We then have :

$$
\mu_{i}=\mu_{i}\left(\|u\|_{L^{\infty}(\Omega)}\right), \mu_{i}<\infty, i=1,2,3, \mu_{2}>0 .
$$

By using the same test functions as in Paragraph 2.2 and 2.3 (this is allowed because we have assumed here that $u \in L^{\infty}(\Omega)$ ) we again obtain the inequality (35), but with $M$ depending also on $\mu_{i}$ now, and with $k$ given by :

$$
k:=\frac{|\nabla u|\left|\varphi_{2}\right|}{\lambda}+\frac{|\nabla u||\nabla \lambda|}{\lambda}+\frac{|\nabla \lambda|\left|\varphi_{1}\right|}{\lambda^{2}}+\frac{\left|\varphi_{1}\right|^{2}}{\lambda^{2}}+|\nabla u|^{\alpha}+\frac{\left|\varphi_{3}\right|}{\lambda} .
$$

Under the assumptions made on $\lambda, \varphi_{i}$ and $\alpha$ we recover the fact that $k \in L^{\beta}(\Omega)$ for some $\beta>1$. The proof can then be completed by following the reasoning presented in Paragraph 2.4. 
We now give some indications concerning the remarks at the end of Paragraph 1.2.

Firstly, if we consider $\lambda \in \mathcal{K}^{\prime}$ instead of $\lambda \in \mathcal{K}$ then the proofs presented previously work. It sufficies to consider the test functions $\varphi$ given by

$$
\varphi=\frac{u-\bar{u}}{\rho} \psi^{2},
$$

instead of (36) and

$$
\varphi=\frac{u}{\rho} \psi^{2}
$$

instead of (43).

If we remove the assumption (13), then, as explained in Section 1, we need not have $H_{0}=W_{0}$. Nevertheless, the first part of Theorem 1 can be established for $W$-solutions by using Proposition 2.6 and Theorem 2.9 in [31]. For the last part we can carry over the arguments presented in the Paragraphs 2.2, 2.3 and 2.5. In fact, the test functions $\varphi$ we have used were always in $W_{0}$ and they are thus admissible for the $W$-formulation.

\section{Additionnal remarks and examples}

In the first paragraph we will present some examples in dimensions two and three of some weights satisfying our assumptions in $\mathcal{K}$ but which are not in $\mathcal{A}_{2}$. In dimension three we present a critical example of a weight in our class $\mathcal{K}$. It is apparently close to satisfying the condition (UE) but we will prove that it does not satisfy (M). Moreover, this weight does not have bounded mean oscillations.

In a second paragraph we will give some remarks concerning the one dimensional case and the case where $\mathcal{A}(x)=\lambda(x) I d$.

\subsection{EXample of Weights in the Class $\mathcal{K}$ BUt not in the CLASS $\mathcal{A}_{2}$.}

We present a first example in dimension two. Let $\Omega$ be the unit disc in $\mathbb{R}^{2}$. We denote by $\Omega^{-}$the inferior half disc. We also consider the sectors $\Lambda_{2}, \Lambda_{0}$ and $\Lambda_{1}$ having a polar angle $\theta$ between the values 0 and 
$\pi / 4, \pi / 4$ and $(3 \pi) / 4,(3 \pi) / 4$ and $\pi$, respectively. We set

$$
\begin{aligned}
& \lambda=r^{-1 / 2} \quad \text { in } \Lambda_{0}, \\
& \lambda=1 \quad \text { in } \Omega^{-},
\end{aligned}
$$

and otherwise we define $\lambda$ by an affine interpolation with respect to $\theta$, that is :

$$
\begin{aligned}
& \lambda=\frac{4}{\pi} \frac{1-\sqrt{r}}{\sqrt{r}} \theta+1 \quad \text { in } \Lambda_{2}, \\
& \lambda=\frac{4}{\pi} \frac{\sqrt{r}-1}{\sqrt{r}} \theta+\frac{4-3 \sqrt{r}}{\sqrt{r}} \text { in } \Lambda_{1} .
\end{aligned}
$$

We can then verify the following :

$$
\begin{aligned}
\lambda & \geq 1 \quad \text { everywhere on } \Omega, \\
\lambda & \in W^{1, s}(\Omega) \text { for each } s<4 / 3, \\
\lambda^{-1 / 2} & \in W^{1,7 / 3}(\Omega) .
\end{aligned}
$$

Consequently, the assumptions (16)-(19) are fulfilled. Moreover, by using the Corollary 4.4 in [40] we can see that $\lambda$ is regular.

It follows that $\lambda$ is in the class $\mathcal{K}$ for which Theorem 1 and Corollary 2 can be applied. Nevertheless $\lambda \notin \mathcal{A}_{2}$.

To see this, we consider the sequence of points $x_{k}=(0,-1 / k)$. For $k$ sufficiently large, the disc $B\left(x_{k}, 2 / k\right)$ is included in $\Omega$ and we have :

$$
\int_{B\left(x_{k}, \frac{2}{k}\right)} \lambda d x \geq \int_{B\left(0, \frac{1}{k}\right) \cap \Lambda_{0}} \lambda d x=\frac{1}{4} \int_{B\left(0, \frac{1}{k}\right)} r^{-1 / 2} d x=\frac{\pi}{3 k^{3 / 2}} .
$$

On the other hand :

$$
\int_{B\left(x_{k}, \frac{1}{k}\right)} \lambda d x=\left|B\left(x_{k}, \frac{1}{k}\right)\right|=\frac{\pi}{k^{2}} .
$$

Hence

$$
\frac{\int_{B\left(x_{k}, \frac{2}{k}\right)} \lambda d x}{\int_{B\left(x_{k}, \frac{1}{k}\right)} \lambda d x} \geq \frac{\sqrt{k}}{3} \rightarrow \infty \quad \text { when } k \rightarrow \infty .
$$

This implies that the measure $\lambda d x$ is not doubling, and thus $\lambda \notin \mathcal{A}_{2}$ (see [19]).

In this situation, Theorem 1 is not a consequence of Theorem 3.3.6 
p135 in [35], and Corollary 2 cannot follow by the results in [11].

We consider now a critical example in dimension three.

Let $\Omega=B\left(0, e^{-4}\right) \subset \mathbb{R}^{3}$. We consider the partition $\Omega=\Omega^{-} \cup \Lambda_{0} \cup \Lambda_{3}$, where :

$$
\begin{aligned}
& \Omega^{-}=\{(x, y, z) \in \Omega: z<0\} \\
& \Lambda_{0}=\{(x, y, z) \in \Omega: \varphi \in(0, \pi / 4)\} \\
& \Lambda_{3}=\{(x, y, z) \in \Omega: \varphi \in(\pi / 4, \pi / 2)\} .
\end{aligned}
$$

Here $\varphi$ denotes the colatitude in spherical coordinates.

We set :

$$
\lambda=\left\{\begin{array}{l}
\ln (-\ln (r)) \quad \text { in } \Lambda_{0}, \\
1 \text { in } \Omega^{-}, \\
\frac{4}{\pi}\left(1-\ln (-\ln (r)) \varphi+2 \ln (-\ln r)-1 \quad \text { in } \Lambda_{3} .\right.
\end{array}\right.
$$

Notice that, on the sector $\Lambda_{3}$ we have defined $\lambda$ by interpolating (with respect to $\varphi$ ) between the values on $\Lambda_{0}$ and on $\Omega^{-}$.

We have :

$$
\begin{aligned}
& \lambda \geq 1 \quad \text { in } \Lambda, \\
& \lambda \in W^{1,3}(\Omega) .
\end{aligned}
$$

Remark that (54) together with (55) implies that $\lambda^{-1 / 2} \in W^{1,3}(\Omega)$. We can also verify that $\sqrt{\lambda} \in H^{1}(\Omega)$, and by using Theorem 3.1 in [15] we can show that $\lambda$ is regular. Hence $\lambda$ is in the class $\mathcal{K}$ for which Theorem 1 and Theorem 3 work.

By appling the same method as in the previous example we can verify that $\lambda \notin \mathcal{A}_{2}$. Notice that here we have $\lambda^{-1} \in L^{\infty}(\Omega)$ and $\lambda \in W^{1,3}(\Omega) \subset$ $\cap_{p \geq 1} L^{p}(\Omega)$. This is a limit case: apparently $\lambda$ is nearly satisfying (UE), nevertheless $\lambda \notin \mathcal{A}_{2}$. Remark also that here Theorem 1 allows us to obtain a weighted higher integrability for the gradient of $u$. Namely, the weak solution of problem $(\mathrm{P})$ satisfies:

$$
\int_{\Omega} \lambda|\nabla u|^{2+\varepsilon}<\infty
$$

for some $\varepsilon>0$.

Finally we can even verify that $\lambda \notin B M O$. In fact (see [34] p218), each BMO function $\psi$ can be written in the form $\psi=c \ln \omega$, with $\omega \in \mathcal{A}_{p}$ and $p>1$. Here $\mathcal{A}_{p}$ denotes the Muckenhoupt class of order $p$ (see [19]). In particular the measure $\omega d x$ is doubling (see [19]). But, $e^{\lambda} d x$ is 
not doubling (use again the same arguments as for $\lambda d x$ ). Consequently $\lambda \notin B M O$, and Theorem 1 cannot be deduced from [36].

\subsection{Some SPecial CASES For Problem $(\mathrm{P})$}

In some particular situations we can obtain the results contained in Theorem 1 and Corollary 2 more easily.

This is true for instance in the one dimensional situation. Let $I=(a, b)$ be a finite interval on the real line. The problem $(\mathrm{P})$ takes the form :

$$
\begin{aligned}
\left(\lambda u^{\prime}\right)^{\prime} & =f \quad \text { in } I, \\
u(a)=u(b) & =0 .
\end{aligned}
$$

If we consider $f \in L^{1}(I)$ then this problem can be solved without using the weighted Sobolev setting, and it suffices to make the assumptions (5)-(6) on $\lambda$. In fact, by a direct integration we explicitly obtain a distributional solution $u$. Namely :

$$
\begin{aligned}
u(x) & =\int_{a}^{x} \frac{1}{\lambda(t)}\left(c_{1}+\int_{a}^{t} f(s) d s\right) d t \\
c_{1} & =\frac{-\int_{a}^{b} \int_{a}^{t} f(s) d s d t}{\int_{a}^{b} \frac{1}{\lambda(t)} d t} .
\end{aligned}
$$

We can see that the weak derivative of $u$ is given by

$$
u^{\prime}(x)=\frac{1}{\lambda(x)}\left(\int_{a}^{x} f(t) d t+c_{1}\right) .
$$

It follows that if $\lambda \in L^{s}(I)$ then $u \in W^{1, s}(I)$. Consequently $u \in$ $\mathcal{C}^{0,(s-1) / s}(\bar{I})$ (see [5] Theorem VIII.2 p.122).

Let now $n \geq 2$, and consider the particular case where $\mathcal{A}(x)=\lambda(x) I d$. Assume that the hypothesis of Theorem 1 is satisfied. By using the first part of Theorem 1 we know that there exists a unique weak solution $u \in H_{0}$ for problem (P). Moreover, in this case we have :

$$
-\Delta u=\underbrace{-\frac{f}{\lambda}}_{:=f_{1} \in W^{-1, p}(\Omega)}+\underbrace{\frac{\nabla u \nabla \lambda}{\lambda}}_{:=f_{2}} .
$$

cor_article.tex; 20/04/2006; $11: 32 ;$ p.22 
By using the assumption (16) we can see that

$$
f_{2}=\lambda^{1 / 2} \nabla u \frac{\nabla \lambda}{\lambda^{3 / 2}} \in L^{\beta}(\Omega),
$$

with $\beta=(2 \sigma) /(2+\sigma)$.

Let us denote by $G$ the inverse of the Laplacian operator on $\Omega$ with homogeneous Dirichlet conditions on $\partial \Omega$, and consider the functions $u_{1}=G\left(f_{1}\right), u_{2}=G\left(f_{2}\right)$. If we assume that $\Omega$ is of class $\mathcal{C}^{2}$ then by using classical regularity results (see for instance [33], Theorem 7.2 p.123 and [5] Theorem IX.25 p.181) we obtain :

$$
u_{1} \in W_{0}^{1, p}(\Omega), \quad u_{2} \in W^{2, \beta}(\Omega) .
$$

By employing the Sobolev imbedding theorem we see that $u_{2} \in W^{1, \beta^{*}}(\Omega)$, with $\beta^{*}=(n \beta) /(n-\beta)$. It follows that for $n=2$ we obtain $\beta^{*}>2$ and we recover the last part of Theorem 1 . When $n>2$ we have to assume that $\sigma>n$ in order to recover in this manner the last result of Theorem 1.

\section{References}

1. Y. A. Alkhutov, V. V. Zhikov: 'On the Hölder continuity of solutions to degenerate elliptic equations', Dokl. Math. 63(3) pp.368-373.

2. J. Björn: 'Poincaré inequalities for powers and products of admissible weights', Ann. Aca. Sci. Fennicae 26 (2001), 175-188.

3. B. V. Boyarskii: 'Generalized solutions of a system of differential equation of the first order of elliptic type with discontinuous coefficients', Math. Sbornik N. S. 43 (1957), 451-503 (Russian).

4. D. Bresch, J. Lemoine, F. Guillen-Gonzalez: 'A note on a degenerate elliptic equation with applications for lakes and seas', Elec. Jour. of Diff. Equa. 2004(42) (2004), 1-13.

5. H. Brezis: 'Analyse fonctionnelle, théorie et applications', Masson, Paris Milan Barcelone (1993).

6. C. Capone, L. Greco, T. Iwaniec: 'Higher integrability via Riesz transforms and interpolation', Nonlinear Analysis 49 (2002) 513-523.

7. S. Clain, R. Touzani: 'A two dimensional stationary induction heating Problem', Math. Mod. Numer. Anal. 31(7) (1997), 845-870.

8. S. Clain, R. Touzani: 'Solution of a two-dimensional stationary induction heating problem without boundedness of the coefficient', Math. Mod. Meth. Appl. Sci. 20 (1997), 759-766.

9. E. Degiorgi: 'Sulla differenziabilità e l'analiticità delle estremali degli integrali multipli regolari', Mem. Acad. Sci. Torino Cl. Sci. Fis. Mat. Nat. 3 (1957), 25-43.

10. L. C. Evans, R. F. Gariepy: 'Measure theory and fine properties of functions', Boca Raton, CRC Press (1992). 
11. E. B. Fabes, E. Kenig, R. P. Serapioni: 'The local regularity of solutions of degenerate elliptic equations', Comm. Part. Diff. Equa. 7(1) (1982), 77-116.

12. B. Franchi, R. P. Serapioni, F. S. Cassano: 'Irregular solutions of linear degenerate ellitpic equations', Potential Analysis 9 (1998), 201-216.

13. T. Iwaniec: 'Projections onto gradient fields and $L^{p}$ estimates for degenerate elliptic operators', Studia Mathematica 75 (1983), 293-312.

14. T. Gallouet, R. Herbin: 'Existence of a solution to a coupled elliptic system', Appl. Math. Lett. 7(2) (1994), 49-55.

15. T. Gallouet, J. Lederer, R. Lewandowski, F. Murat, L. Tartar: 'On a turbulent system with unbounded eddy viscosities', Nonlinear Analysis 52 (2003), 10511068.

16. M. Giaquinta, M. Struwe: 'On partial regularity of weak solutions for nonlinear parabolic systems', Math. Z. 179 (1982), 437-451.

17. M. Giaquinta: 'Multiple integrals in the calculus of variations and nonlinear elliptic systems', Annals of Mathematics Studies 105, Princeton University Press, Princeton (1983).

18. F. W. Ghering: 'The $L^{p}$-integrability of the partial derivatives of quasiconformal mapping', Acta Mathematica 130 (1973), 256-277.

19. J. Heinonen, T. Kilpelainen, O. Martio: 'Nonlinear potential theory of degenerate elliptic equations', Oxford Mathematical Monographs, Clarendon Press (1993).

20. T. Kilpelainen: 'Smooth approximation in weighted Sobolev spaces', Comm. Math. Univ. Carolinae 38(1) (1997), 29-35.

21. J. Kinnunen: 'Higher integrability with weights', Ann. Aca. Sci. Fennicae, Ser. A.I Math. 19 (1994), 355-366.

22. J. Kinnunen: 'Higher integrability for parabolic systems of $p$-Laplacien type', Duke Math. J. 102(2) (2000), 253-271

23. R. Lewandowski: 'Analyse mathématique et océanographie', Masson Paris, (1997).

24. N. G. Meyers: 'An $L^{p}$ estimate for the gradient of solutions of second order elliptic divergence equations', Ann. Scu. Norm. Sup. Pisa 17 (1963), 189-206.

25. N. G. Meyers, A. Elcrat: 'Some results on regularity for solutions of non-linear elliptic systems and quasi-regular functions', Duke Math. J. 42 (1975), 121-136.

26. M. Mastylo, M. Milman: 'A new approach to Gehring's lemma', Indiana Univ. Math. Jour. 49(2) (2000), 655-679.

27. J. Moser: 'A new proof of De Giorgi's theorem concerning the regularity problem for elliptic differential equations', Comm. Pure Appl. Math. 13 (1960), $457-468$.

28. J. Moser: 'On Harnack's theorem for elliptic differential equations', Comm. Pure Appl. Math. 14 (1961), 577-591.

29. M. K. V. Murthy, G. Stampacchia: 'Boundary value problems for some degenerate-elliptic operators', Ann. Math. Pura. Appl. IV 80 (1968), 1-122.

30. J. Nash: 'Continuity of solutions of parabolic and elliptic equations', Amer. J. Math. 80 (1958), 931-954.

31. F. Serra Cassano: 'On the local boundedness of certain solutions for a class of degenerate elliptic equations', Bollettino U.M.I (7)10-B (1996), 651-680.

32. P. Shi, S. Wright: 'Higher integrability of the gradient in linear elasticity', Math. Ann. 299(3) (1994), 435-448.

33. C. G. Simader: 'On Dirichlets's boundary value Problem', Lectures notes in mathematics 268, Springer Berlin (1972). 
34. E. M. Stein: 'Harmonic analysis. Real-variable methods, orthogonality and oscillatory integrals', Princeton Mathematical Series 42, Princeton University Press (1993).

35. E. W. Stredulinski: 'Weighted inequalities and degenerate elliptic partial differential equations', Lecture notes in mathematics 1074, Springer Berlin (1984).

36. B. Stroffolini: 'Elliptic systems of PDE with BMO-coefficients', Potential Analysis 15 (2001), 285-299.

37. N. S. Trudinger: 'On the regularity of generalized solutions of linear, nonuniformly elliptic equations', Arch. Ration. Mech. Anal. 42 (1971), 50-62.

38. N. S. Trudinger: 'Linear elliptic operators with mesurable coefficients', Ann. Sc. Norm. Super. Pisa, Sci. Fis. Mat., III.27 (1973), 265-308.

39. C. Yemin: 'Regularity of solutions to the Dirichlet problem for degenerate elliptic equation', Chin. Ann. Math. 24B(4) (2003), 529-540.

40. V. V. Zhikov: 'Weighted Sobolev spaces', Sbornik Mathematics 189(8) (1998), $1139-1170$.

41. V. V. Zhikov: 'To the problem of passage to the limit in divergent nonuniformly elliptic equations', Functional Analysis and Its Applications 35(1) (2001), 1933. 